\title{
DEL ESTADO DESPÓTICO AL COMUNISMO PRIMITIVO
}

\section{FROM HYDRAULIC DESPOTISM TO PRIMITIVE COMMUNISM}

\author{
Jacinta Palerm Viqueira \\ Colegio de Postgraduados (México)
}

\section{RESUMEN}

La discusión en torno a la hipótesis hidráulica ha generado interesantes estudios sobre la autogestión de sistemas hidráulicos; no obstante se ha hecho poco por diferenciar los tipos de autogestión. En este ensayo se propone, además de una tipología por el tipo de administración: por personal técnico contratado en cadena de mando o por los mismos regantes, una tipología por el tipo de productores.

Palabras clave: Sistemas de riego, auto-gobierno, administración burocrática, administración no-burocrática, tipo de productores

\begin{abstract}
The debate concerning the hydraulic hypothesis has produced interesting studies on the self-governance of irrigation systems. However, little has been done to classify the different types of self-governance. In addition to a typology based on management type, that is by hired technical staff in a command chain or the irrigators themselves, this paper proposes a typography based on the characteristics of the farmers.
\end{abstract}

Keywords: Irrigation system, self-governance, bureaucratic management, non-bureaucratic management, farmer typology 


\section{INTRODUCCIÓN}

Los diversos estudios que han sometido a prueba la llamada hipótesis hidráulica, han puesto menos atención a caracterizar la diversidad del auto-gobierno.

Hemos propuesto que tomar en cuenta el tipo de administración: burocrática o no-burocrática ofrece posibilidades de análisis interesantes; los grandes sistemas efectivamente se correlacionan con un desarrollo de burocracia agro-gerencial (PALERM-VIQUEIRA, 2006). Otro asunto es el poder que concentren esas burocracias y como señala Wittfogel, en sociedades pluricéntricas, las burocracias hidráulicas, ejemplificado con los casos del Tennesse Valley Authority (TVA) en Estados Unidos y el proyecto Snowy Mountain en Australia, no son miembros de la burocracia gobernante, sino de una burocracia controlada (WITtFogel, 1970, p. 6)'. También hace referencia a los casos de obras hidráulicas de Holanda, el valle de Po, Venecia y de los mormones en Utah-donde las grandes obras hidráulicas no desarrollaron un orden hidráulico (WitTFOGel, 1957, p. 12)2.

Hemos propuesto que tomar en cuenta el marco jurídico para las organizaciones autogestivas puede ser un elemento importante para sopesar la capacidad de negociación con el Estado, así como la consolidación y visibilidad de las organizaciones (Palerm-Viqueira, 2010a). La consolidación de organizaciones y su capacidad negociadora con el Estado que propone Maass (1978), parece vinculada al marco jurídico. Su ausencia, entre otros

1 "What I have been saying about traditional China is not valid for multicentred societies. In private-property.based industrial societies big hydraulic works frequently fulfil different functions, among them and especially the generation of electric power. But even when they also fulfil tasks of flood control (as does the Tennessee Valley Authority in the USA) or of irrigation (as does the Snowy Mountain Scheme in Australia), they are not members of a ruling bureaucracy, but of a controlled bureaucracy." (WitTFOGEL, 1970, p. 6).

2 "Large enterprises of water control will create no hydraulic order, if they are part of a wider nonhydraulic nexus. The water works of the Po Plain, of Venice, and of the Netherlands modified regional conditions; but neither Northern Italy nor Holland developed a hydraulic system of government and property." (Wittfogel, 1957, p. 12). 
factores, podría explicar la existencia de lo que Ruf $(2000,2011)$ denomina un despotismo occidental así como la expansión de la administración por el Estado desplazando a las organizaciones de regantes que se presentó en el siglo XX en México (PalerM-VIQUeIRA, 2009). Sin embargo, como hemos presentado en los estudios de caso reunidos en Antología sobre riego: Instituciones para la gestión del agua: vernáculas, alegales e informales (2013) aparentemente estamos ante un problema de visibilidad de las organizaciones y no de su inexistencia. Los casos se refieren a organizaciones compuestas por productores que son gente común: pequeños agricultores y la existencia de comunidades organizadas parece ser un elemento detonador de la auto-organización para gestionar el conjunto de sistemas hidráulicos multi-comunitarios.

Es esta vertiente que proponemos explorar: la caracterización basada en el tipo de productores.

\section{LA GENTE COMÚN}

Erickson (2006), autor de uno de los capítulos de Agricultural Stategies, critica lo que llama el enfoque neo-Wittfogeliano o de economía política, que señala que existe algún tipo de relación causal entre intensificación de la agricultura y origen del Estado. El enfoque neo-Wittfogeliano también incorpora un concepto que ya brevemente había tocado G. Childe (1964) -y que también critica Erickson- el requerimiento de mecanismos de coerción para la producción (y entrega) de excedentes. En los análisis de Chayanov (1985) y de Sahlins (1977) sobre el modo doméstico de producción las unidades domésticas despliegan trabajo para satisfacer sus necesidades de consumo y, una vez cubiertas, la inversión de trabajo cesa y, por lo tanto, no van a producir excedentes.

Erickson (2006: 338-340) argumenta que se presta poca atención a las capacidades de los campesinos de manejar sistemas agrícolas complejos intensivos sin Estado. Hace referencia a diversas etnografías, entre ellas los estudios de Lansing (1991) sobre Bali y los de Glick (1970) sobre la huerta de Valencia como ejemplos de coordinación de sistemas agrícolas intensivos o sin intervención del Estado. Sin embargo, el estudio de Lansing sobre Bali se refiere al gobierno coordinado de una cuenca que riega unas 8,600 ha, donde cada sistema riega entre 30 y 150 hectáreas (Lansing, 1991: 42, 46, 118-119); las 100.000 hectáreas que refiere Mabry (2000: 291) para Bali representan más bien un continuo geográfico de pequeños regadíos. Los sistemas de la huerta de Valencia que estudia Glick no rebasan las 7.000 hectáreas, aunque hay un sistema en la huerta que riega 20.000 hectáreas; por otro lado las grandes presas de almacenamiento que, desde 
principios del siglo $X X$, regulan el agua de los ríos son administrados por una institución del Estado (GLICK, 1970; PIMentel-Equinua \& PalerM-VIQUeIRA, 2009). Las dimensiones del regadío de Bali o de la huerta de Valencia pueden compararse con las de una obra de cabecera en China, construida en 200 a.C. y que sirvió para regar inicialmente unas 200.000 ha., el sistema, todavía en funcionamiento, riega actualmente unas 700.000 hectáreas (JONES, 1954; VALE, 1905; WILLMOTt, 1989; Yongtang, 1988).

Otras ejemplos de etnografías que refiere ERICKSON (2006: 338) como aquellas de TRAWICK (2001) y Gelles (1984) también tratan sobre sistemas pequeños o medianos; mientras que los trabajos de MABRY (1996) señalan claramente diferencias entre pequeña y grande irrigación por las demandas administrativas e ingenieriles de los sistemas de riego y, en el caso de los trabajos de HUNT (1997), toma en cuenta únicamente el tipo de gobierno y no el tipo de administración (burocrático o no burocrático) (PALERM-VIQUeIRA, 2006).

Erikson pone el acento en la capacidad de la gente común, sin élites, de administrar sistemas agrícolas complejos. Ello significa no solo la ausencia de una burocracia agrogerencial, sino también una producción por "gente común" es decir agricultores campesinos que no contratan o utilizan mano de obra extra familiar, de otra manera estaríamos hablando de elites.

Es pertinente, en seguimiento al reproche que hace Erickson de que no se pone atención a la capacidad de la gente común de administrar sistemas agrícolas complejos, tomar nota del contraste que hacen algunos autores al comparar regiones con tradición jurídica de auto-gobierno. Se destacan diferencias entre el control de las organizaciones por pequeños agricultores (campesinos) o por inversionistas citadinos. Los contrastes son entre Holanda y Lombardía (en general el valle del Po, donde se ubica la región de Lombardía), así como entre Flandes (la parte flamenca de Bélgica) y Holanda.

El valle del Po, así como Holanda son ejemplos de inversión y gestión no centralizada y no estatal de obra de control del agua. En el valle de Po hay una superficie de riego superior al millón de hectáreas, continuo de regadío que abarca desde las ciudades de Turín y Milán hasta la ciudad de Ferrara; además forma un continuo geográfico con la región del Veneto, rica en obras de control del agua. Por su parte, la llanura costera entre Frisia y Pas de Calais, donde destaca Holanda, es un continuo de obras de control de agua con fines de drenaje.

En el caso de Holanda, la región es marginal en la época medieval, los señores feudales otorgaban permisos para asentarse $y$, desde un inicio, los campesinos son libres. Como dice un autor (VRIES, 1973) es una región naci- 
da libre. Las asociaciones para diques y drenajes son instituciones campesinas, Fockema (1952), el gran estudioso de estas instituciones en la época medieval las asemeja a las comunas urbanas (ver también KAIJSER, 2002; VRIES, 1973).

Las tareas en torno a los diques y drenajes son, en un inicio, de los consejos aldeanos y, posteriormente, por la complejidad del manejo y por rebasar el ámbito comunitario, se crean instituciones especializadas locales. En 1169, el conde Felipe de Alsacia crea los primeros water ring. Las primeras asociaciones regionales aparentemente recibieron un impulso de los gobernantes, el conde nombraba al presidente del consejo de la asociación: el dijkgraaf (Borger \& Ligtendag, 1998; FOCKEMA ANDREAE, 1952; KaIJSER, 2002; TeBraKe, 2002; VRIES, 1973). Entre fines del siglo XV y el XVI aparecen asociaciones regionales más amplias que, según Kaijser (2002: 529) cubrían espacios que iban de 10.000 a 40.000 hectáreas.

Sobre el desarrollo de una burocracia agrogerencial en estas organizaciones controladas por campesinos tenemos información limitada, pero en el siglo XX, a partir de la década de 1970, la política pública holandesa obliga a la unificación de asociaciones para alcanzar economías de escala y contratar personal profesional (PALERM-VIQUEIRA, 2010b).

En Lombardía, como en todo el valle de Po y a diferencia de Holanda, el dominio pasó de los señores feudales a los citadinos interesados en controlar el entorno agrícola; solo en las montañas en las vertientes del río Po hay comunidades campesinas con control sobre la tierra y el agua. En Flandes, también a diferencia de Holanda, la inversión citadina en el agricultura es temprana y desplaza el control de las organizaciones de diques y drenajes de los campesinos a inversionistas citadinos y a las ciudades (CURTIS \& CAMPOPIANO, 2013; Curtis, 2012; Hall, 1886; PalerM-VIQUeIRA, 2010b; SIRRY, 1902; SMITH, 1852, 1855; SOENS, 2011, 2013).

Con este contraste se pretende señalar que la ausencia de Estado en la administración de los sistemas de riego y obras de control del agua y la existencia de auto-gobierno no implica una gestión sin jerarquías. En algunos casos, como en el valle del Po y Flandes, el control estuvo en manos de las elites citadinas comerciales y no de la gente común: los pequeños agricultores.

\section{EL COMUNISMO PRIMITIVO}

La gestión por gente común, por pequeños agricultores se ilustra de manera ejemplar con los estudios realizados en el siglo XIX sobre las llamadas comunidades aldeanas. Esos estudios han tomado nueva relevancia gracias a un nuevo enfoque que propone que hay una tercera vía para la gestión 
de recursos de uso común distinta a la gestión por el Estado o por el mercado y que consiste en señalar las capacidades autogestivas de regantes y otros usuarios de recursos de uso común (pesca, bosque, pastizales, etc.). El enfoque se desarrolla como parte de la llamada nueva economía institucional (ОлтROM, 2011). El impacto ha sido significativo, la interpretación de la evidencia sobre las comunidades aldeanas (o comunidades organizadas campesinas) se ha modificado.

En el siglo XIX se realizaron estudios sobre las comunidades aldeanas ${ }^{3}$, por ejemplo: Haxthausen, [1847-1852] (1856); Maine, [1861] (1908); Laveleye, [1874] (1878), entre otros. La comunidad aldeana se interpretó como un arcaísmo de una etapa anterior. Marx y Engels darían la denominación de comunismo primitivo a esta etapa ${ }^{4}$, incluso los populistas rusos sugirieron la posibilidad de su rescate para el socialismo 5 .

3 Hacia mediados del siglo XX, se incorpora el, en mi opinión, poco afortunado, aporte de Wolf (1957, 1971), al análisis de la comunidad campesina organizada; sigue el esquema analítico decimonónico: una etapa evolutiva que, con el progreso, debía desaparecer.

4 Como segunda nota a pie de página al Manifiesto Comunista, (Engels, 1888): "In 1847, the pre-history of society, the social organization existing previous to recorded history, [was] all but unknown. Since then, August von Haxthausen (1792-1866) discovered common ownership of land in Russia, Georg Ludwig von Maurer proved it to be the social foundation from which all Teutonic races started in history, and, by and by, village communities were found to be, or to have been, the primitive form of society everywhere from India to Ireland. The inner organization of this primitive communistic society was laid bare, in its typical form, by Lewis Henry Morgan's (1818-1861) crowning discovery of the true nature of the gens and its relation to the tribe. With the dissolution of the primeval communities, society begins to be differentiated into separate and finally antagonistic classes. I have attempted to retrace this dissolution in The Origin of the Family, Private Property, and the State, second edition, Stuttgart, 1886."

5 Los populistas rusos propusieron que la comunidad campesina rusa (obschina o mir), como una forma de comunismo primitivo, podía aportar a la nueva sociedad. Marx, a partir de la interacción con populistas rusos, entre ellos Vera Sassulitch quien le interroga sobre el papel de la comunidad campesina rusa, aprende ruso, lee sobre los campesinos rusos y aporta una respuesta ambigua y cambiante. Por ejemplo, "El análisis presentado en el capital no da, pues, razones, en pro ni en contra de la vitalidad de la comuna rural, pero el estudio especial que de ella he hecho, y cuyos materiales he buscado en las fuentes originales, me ha convencido de que esta comuna es el punto de apoyo de la regeneración social en Rusia ..." (MarX, Edición francesa de El Capital, Cap. xxXII (MarX \& Engels, 1980) 
En la nueva perspectiva sobre las comunidades aldeanas destaca el trabajo de Wade: Village Republics (Repúblicas Aldeanas). WADE (1988) propone la existencia de comunidades organizadas a partir del regadío o del manejo de otros recursos escasos, argumentando que la organización comunitaria es una respuesta económicamente ventajosa frente a la escasez de recursos críticos a la sobrevivencia. La organización comunitaria surge de la puesta en marcha de actividades colectivas que comprenden desde la vigilancia de cultivos hasta la administración del regadío.

La interpretación materialista de Wade tiene especial sustento al demostrar que en una misma región cultural "... las variaciones de escasez y riesgo en la esfera agrícola vital explican mucha de la variación que se encuentra en la organización de las aldeas ..." (WADE, 1988:1). En consonancia con la propuesta de Wade, estudios de caso en México muestran que comunidades cercanas entre si, tienen una mayor o menor organizativa comunitaria dependiendo de la escasez o abundancia de agua de riego (Palerm-Viqueira, Pimentel-Equihua, \& Salcedo, 2000).

Como antecedente al análisis de Wade que vincula la organización comunitaria con aspectos materiales, está el análisis del historiador Marc Bloch sobre las bases materiales de las comunidades organizadas de Europa. BıOCH (1978) señala el estrecho vínculo entre comunidades organizadas, tipo de suelo, uso de la carruca (un tipo de arado) y lo que los ingleses llaman "open fields" (ver también WHITE, 1973). Sin embargo el estudio de Bloch se acota a este caso en particular.

De manera similar antropólogos mesoamericanistas y andinistas habían propuesto un vínculo entre la base material de riego y comunidad organizada, sin embargo, nuevamente se acotaba a esos casos y no explicaba, como bien señala Gelles (1984: 20) la existencia de organización en comunidades sin riego (MILloN, Hall, \& DIAZ, 1997: 122; PALERM, 1980; VIQUeIRA \& PALERM, 1954).

La comunidad aldeana o la comunidad organizada es una respuesta institucional a aspectos económicos; a su vez la organización implica el desarrollo de la esfera política, sin embargo no implica jerarquías o elites.

En las comunidades campesinas organizadas hay un desarrollo de la esfera de lo público, incluso sugiere Wade (1988: XI, mi traducción)

"Si, como algunos politólogos, vemos las bases del Estado en una conjunción de contrato y coerción, y si pensamos que los primeros Estados representan un estadio relativamente avanzado de evolución de lo público en comunidades locales, podemos, a partir de cómo se sustenta la combinación de contrato y coerción en las aldeas 
Indias de nuestros días, buscar nuevas luces de cómo emergió en las comunidades agrícolas de los Estados prístinos."

En el mismo sentido, MABRY (2007) plantea que con las primeras obras de pequeño riego en árido-América, en comunidades de 70- 175 personas hay un desarrollo de la esfera de lo público -pero, tal y como insiste, no-jerárquica. Las bandas de cazadores recolectores transitan al sedentarismo y aparece como novedad por un lado la propiedad individual o de la unidad doméstica y por otro la propiedad corporada. La pequeña obra de riego es una propiedad corporada del conjunto de unidades domésticas y las unidades domésticas tienen derechos y obligaciones hacia la propiedad corporada, por ejemplo derecho al agua y obligación de cumplir faenas de trabajo. Se desarrolla una cooperación y toma de decisiones colectivas sobre la propiedad corporada.

Es posible denominar esta vertiente de la organización social en torno al riego (o al manejo de otros recursos) como la vertiente anarquista de la gestión: igualitaria, sin jerarquías.

\section{El Estado despótico}

La organización social en torno al control del agua para la agricultura, señala un continuo con dos extremos. En un extremo están los casos caracterizados por una administración no jerárquica y basada en sistemas de conocimiento locales. En el otro extremo están los casos caracterizados por una administración a cargo de una burocracia agrogerencial, una propuesta aunada a la evolución socio-cultural de la humanidad, en específico al origen de las primeras civilizaciones. En este continum existen una diversidad de tipos, hemos caracterizado la presencia o ausencia de burocracia, aún con auto-gobierno y la caracterización de los productores, como pequeños o grandes agricultores. En seguida vamos a recordar los antecedentes del interés en el impacto del regadío sobre la sociedad para discutir la propuesta de vínculo entre regadío y origen de la civilización.

En las primeras décadas del siglo XX, Gordon Childe propone que en el registro arqueológico hay dos grandes revoluciones, la revolución del neolítico con el invento de la agricultura y la revolución urbana con la aparición de las primeras civilizaciones. Hay unas pocas civilizaciones que se distinguen por ser primigenias, entre ellas Mesoamérica, la región Andina, Egipto, Mesopotamia, el valle del Indus, China. Otras civilizaciones, secundarias, surgieron a partir del contacto y acumulación presente en las civilizaciones primigenias.

Childe, a partir del registro arqueológico, propone que fue a partir de 
la agricultura de riego que se pudo producir un excedente social del cual se apropió una pequeña elite. Es con la producción y apropiación del excedente social que fue posible sostener a una población de especialistas que no eran productores directos. Childe señala a un conjunto de factores por los cuales la revolución urbana se basa en la agricultura de riego.

La agricultura de riego va asociada o posibilita una alta densidad de población sedentaria; en contraste, la agricultura de temporal es necesariamente itinerante y los asentamientos son pequeños y dispersos; ello es relevante ya que la concentración o dispersión de población es un aspecto crítico en un contexto de limitaciones tecnológicas para el transporte de la producción agrícola.

En la agricultura de riego, la construcción y mantenimiento de canales de riego son tareas sociales y la comunidad en su conjunto debe asignar el agua obtenida por el esfuerzo colectivo a los individuos. Adicionalmente, el control del agua puso en manos de la sociedad una fuerza potente de sanciones. En cambio, señala Childe, los agricultores y pescadores del Egeo se beneficiaron de la acumulación de riqueza en Egipto y Mesopotamia sin tener que someterse al mismo grado de unificación política y división de clases (CHILDE, 1950: 5-6, 8; 1954: 13, 31, 38, 39, 91; 1950, 1958: 15, 344, 361, 1964: 39). ${ }^{6}$

La importancia de la producción de excedentes sociales como base para la civilización explica el interés de los antropólogos por los sistemas agrícolas y, en su caso, las obras hidráulicas. Los avances en el estudio de la civilización Maya ilustran bien los alcances del análisis detallado de estos aspectos, desde el supuesto de la excepcionalidad de la civilización Maya al basarse en una agricultura de tumba, roza y quema hasta los resultados de investigación s más recientes sobre existencia de cultivos intensivos y de obras hidráulicas (DUMOND, 1961; LUCERO \& FASH, 2006; TURNER II, 1981).

Wittfogel, a diferencia de Childe, tiene como punto de partida la historia. En su investigación sobre las características del Estado burocrático chino que concentraba un enorme poder, propone que la base material corresponde a la gran obra hidráulica (para riego o control de inundación).

6 "The digging and maintenance of irrigation channels are social tasks even more than the construction of defensive ramparts or the laying out of streets. The community as a whole must apportion to individual users the water thus canalized by collective effort. Now control of water puts in society's hands a potent force to supplement supernatural sanctions. Society can exclude from access to the channels recalcitrant who will not conform to rules of conduct generally approved. Banishment in an arid zone is a more drastic penalty than in a temperate or tropical clime where land and water are still relatively abundant." (CHILDE, 1964: 39). 
Señala el requerimiento de un aparato organizativo para la administración de la construcción, operación y mantenimiento de obra hidráulica, sea esta pequeña (hidro-agricultura) o grande, en este último caso con el requerimiento adicional de una burocracia agro-gerencial para la administración de la construcción, operación y mantenimiento de la gran obra hidráulica.

El punto central de su argumentación es que la administración (de recursos críticos, como el agua) da poder $y$, en la antigüedad, el aparato organizativo encabezado por una burocracia agro-gerencial, lleva a la conformación de un Estado burocrático despótico.

"The effective management of these works involves an organizational web which covers either the whole, or at least the dynamic core, of the country's population. In consequence, those who control this network are uniquely prepared to wield supreme political power.

(...) No matter whether traditionally nonhydraulic leaders initiated or seized the incipient hydraulic "apparatus," or whether the masters of this apparatus became the motive force behind all important public functions, there can be no doubt that in all these cases the resulting regime was decisively shaped by the leadership and social control required by hydraulic agriculture." (WITTFOGEL, 1957: 27)

Sin embargo, señala, en sociedades pluricéntricas, las burocracias hidráulicas, ejemplificado con los casos del Tennesse Valley Authority (TVA) en Estados Unidos y el proyecto Snowy Mountain en Australia, no son miembros de la burocracia gobernante, sino de una burocracia controlada (Wittfogel, 1970: 6). También hace referencia a los casos de obras hidráulicas en Holanda, el valle de Po, Venecia y por los mormones en Utah, donde las grandes obras hidráulicas no desarrollan un orden hidráulico. (WittFogel, 1957: 12).

En conclusión, aunque los sistemas más grandes y más complejos aparentemente dan lugar a un aparato agrogerencial, es decir dan lugar a jerarquías y burocracias, otro asunto es si dan lugar a una burocracia despótica, que controla en lugar de ser controlada.

La exploración sobre cómo los regantes (el gobierno de los regantes) controla a la burocracia agrogerencial parece entonces una pregunta interesante. Misma interrogante que se ha realizado para empresas, incluyendo a las empresas cooperativas (BAtallle -Chedotel \& Huntzinger, 2004; Puentes Poyatos, Velasco Gámez, \& Vilar Hernández, 2009; Ruiz, 2006; Spear, 2004). 


\section{Conclusiones}

En la época actual se pueden hacer las siguientes caracterizaciones en torno a la gestión de los sistemas de riego:

- Gobierno por los mismos regantes o por alguna institución del Estado o por grandes empresas.

- Administración de tipo burocrática o no burocrática.

- Los regantes son pequeños o grandes agricultores.

El autogobierno con administración no burocrática es característico de regantes que son pequeños agricultores.

Sería interesante analizar si la administración por alguna institución del Estado mas bien se correlaciona con regantes que son pequeños agricultores; mientras que el autogobierno con administración burocrática (como en Lombardía) más bien se correlacione con grandes agricultores.

\section{Referencias}

Batallle-Chedotel, F., \& Huntzinger, F. (2004): "Directivos y gobierno de las cooperativas obreras de producción. Un estudio exploratorio sobre diez cooperativas francesas". Revista de Economía Pública, Social Y Cooperati$v a, 48, \mathrm{pp} 79-98$.

BıOCH, M. (1978). [1931]: La historia rural francesa: Caracteres originales. Barcelona: Editorial Crítica-Grijalbo.

Borger, G., \& LigtendaG, W. A. (1998): "The role of water in the development of The Netherlands -a historical perspective". Journal of Coastal Conservation, 4, pp 109-114.

Chayanov, A. V. (1985) [1924]: La organización de la unidad económica campesina. Argentina: Ediciones Nueva Visión.

CHILDE, G. V. (1950): "The Urban Revolution". Town Planning Review, 21, pp 3-17. Retrieved from http://faculty.washington.edu/plape/citiesautll/ readings/Childe-urban\%20revolution\%201950.pdf

CHILE, G. V. (1958) [1926 revised edition 1958]: The Dawn of European Civilization. New York: Alfred A. Knopf. Retrieved from http://rbedrosian. com/Downloads/Childe_DawnofEuropCiv.pdf

CHILDE, G. V. (1964) [1942, revised edition 1954]: What Happened in History. Baltimore, Md.: Penguin Book. Retrieved from http://gyanpedia.in/Portals/0/Toys\%20from\%20Trash/Resources/books/gordonchilde.pdf

CURTIS, D. R. (2012): Pre-industrial societies and strategies for the exploitation of resources. A theoretical framework for understanding why some settlements are resilient and some settlements are vulnerable to crisis (PhD). 
Utrecht University, Research Institute for History and Culture.

Curtis, D. R., \& Campopiano, M. (2013): "Medieval land reclamation and the creation of new societies: comparing Holland and the Po Valley, c.800ec.1500". Journal of Historical Geography, pp 1-16. doi:http://dx. doi.org/10.1016/i.jhg.2013.10.004

DumOND, D. E. (1961): "Swidden Agriculture and the Rise of Maya Civilization". Southwestern Journal of Anthropology, 17 (4), pp 301-316. Retrieved from http://www.jstor.org/stable/3628942.

ENGELS, F. (1888): nota a pie de página al Manifiesto comunista de K. Marx. In Manifiesto comunista. Retrieved from http://www.marxists.org/archive/ marx/works/1859/critique-pol-economy/preface.htm

ERICKSON, C. L. (2006): "Intensification, Political Economy, and the Farming Community"; In Defense Of A Bottom-Up Perspective Of The Past. In J. Marcus \& C. Stanish (Eds.), Agricultural Strategies, pp 334-363. Los Angeles: Cotsen Institute.

Fockema Andreae, S. J. (1952): "Embanking and Drainage Authorities in the Netherlands during the Middle Ages". Speculum, 27 (2), pp 158-167.

Gelles, P. (1984): Agua, faenas y organización comunal en los Andes: El caso de San Pedro de Casta (Tesis Maestría en Antropología). Pontificia Universidad Católica del Perú, Perú.

Guck, T. (1970): Irrigation and society in Medieval Valencia. Cambridge, Mass.: Harvard University Press.

Hall, W. H. (1886): Irrigation Development. History, Customs, Laws and administrative systems relationg to irrigation, water-courses, and waters in France, Italy and Spain. Sacramento: State Office, James J. Ayers, Supt. State Printing. Retrieved from https://archive.org/details/cu31924003976754

Haxthausen, A. B. von. (1856) [1847-1852]: The Russian empire, its people, institutions and resources. (R. Farie, Trans.) (Vol.1). London: Chapman and Hall. Retrieved from https://archive.org/details/russianempireit00farigoog HUNt, R. C. (1997). [1988]: "Sistemas de riego por canales: tamaño del sistema y estructura de la autoridad". In J. Palerm-Viqueira (Ed.), Antología sobre pequeño riego (Vol. 1, pp. 185-219). México: Colegio de Postgraduados.

JONES, F. (1954): Tukiangyien: China's Ancient Irrigation System. Geographical Review, 44 (4), pp 543-559.

KAIJSER, A. (2002): "System building from below. Institutional change in Dutch water control systems". Technology and Culture, 43, pp 521-548.

LANSING, S. (1991): Priests and programmers. Technologies of power in the 
engineered landscape of Bali. Princeton, New Jersey: Princeton University Press.

Laveleye, É. L. V. de (1878) [1874]: Primitive Property. (G. R. L. Marriott, Trans.). London: McMillan and Co. Retrieved from http://www.archive.org/details/ primitiveproper01leslgoog

LUCERO, L. J. \& FASH, B. W. (Eds.) (2006): Precolumbian Water Management: Ideology, Ritual and Power. Tucson: University of Arizona Press. Retrieved from http://www.anthro.illinois.edu/faculty/lucero/documents/6and7-LuceroUncorrected.pdf

MAAss, A., \& ANDerson, R. L. (1978): ... and the desert shall rejoice. Conflict, growth and justice in arid environments. Cambridge: The MIT Press.

MABRY, J. B. (Ed.) (1996): Canals and Communities. Small Scale Irrigation Systems. Tucson: University of Arizona Press.

MABRY, J. B. (2000): "Wittfogel was half right: The ethnology of consensual and nonconsensual hierarchies in irrigation management". In M. W. Diehl (Ed.), Hierarchies in Action: Cui Bono?, pp 284-294. Carbondale: Center for Archaeological Investigation, Southern Illinois University.

MABRY, J. B. (2007): "Chap. 12 Irrigation, short-term sedentism, and corporate organization during the San Pedro phase". In J. B. Mabry (Ed.), Las Capas: Early Irrigation and Sedentism in a Southwestern Floodplain, Draft Report, pp 293-327. Tucson, Arizona: Anthropological Papers No. 28, Center for Desert Archaeology. Retrieved from http://www.cdarc.org/pages/library/ ap28-review/

MaINE, H. J. S. (1908) [1861]: Ancient Law: Its Connection with the Early History of Society, and lts Relation to Modern Ideas. London: John Murray. Marx, C., \& Engels, F. (1980): Escritos sobre Rusia, II El porvenir de la comuna rural rusa. Cuadernos de Pasado y Presente.

Millon, R., Hall, C., \& Diaz, M. (1997) [1962]: "El conflicto en el sistema de riego del Teotihuacan moderno". In Antología sobre pequeño riego, pp 89-146. México: Colegio de Postgraduados.

Ostrom, E. (2011) [1990]: El gobierno de los bienes comunes. La evolución de las instituciones de acción colectiva. México: Fondo de Cultura Económica/SEMARNAP.

Palerm, A. (1980) [1972]: "Aspectos Agrícolas del Desarrollo de la Civilización Prehispánica en Mesoamérica". In A. Palerm \& E. Wolf, Agricultura y Civilización en Mesoamérica, pp 9-29. México: SEP Diana.

PalerM-VIQueIRA, J. (2006): "Self-Management of Irrigation Systems, a Typology: The Mexican Case". Mexican Studies/Estudios Mexicanos, 22 (2), pp 361-385. 
Palerm-Viqueira, J. (2009): "Distritos de riego en México, algunos mitos". In J. Palerm-Viqueira \& T. Martínez Saldaña (Eds.), Aventuras con el agua. La administración del agua de riego: historia y teoría, pp 277-328. México: Colegio de Postgraduados.

PALERM-VIQUeIRA, J. (2010a): "Comparative history of irrigation water management, from the sixteenth to twentieth centuries: Spain, Mexico, Chile, Mendoza (Argentina) and Perú". Water Policy, 12, pp 779-797.

Palerm-Viqueira, J. (2010b): "La utilidad de estudios de larga duración al análisis del impacto del regadío sobre la organización social. El caso de las sociedades de drenaje y diques de Holanda llamados waterschappen (versión corregida)". In D. Murillo \& J. Palerm-Viqueira (Eds.), Presented at the Primer Congreso Red de Investigadores Sociales Sobre Agua, Jiutepec, Morelos: Instituto Mexicano de Tecnología del Agua. Retrieved from http:// redissa.hostei.com/rissa/Mesas_2010.htm

Palerm-Viqueira, J. \& Martínez-Saldaña, T. (Eds.) (2013): Antología sobre riego: Instituciones para la gestión del agua: vernáculas, alegales e informales. Mexico: Biblioteca básica de agricultura, Colegio de Postgraduados/ Grupo Mundi-Prensa.

Palerm-Viqueira, J., Pimentel-Equihua, J. L. \& Salcedo, I. (2000): “Organización diferencial y escasez de agua: caso río Cuautla, Morelos". In J. Palerm-Viqueira \& T. Martínez Saldaña (Eds.), Antología sobre pequeño riego. Organizaciones autogestivas, Vol. 2, pp 73-96. Colegio de Postgraduados/ Plaza y Valdés.

Pimentel-Equihua, J. L., \& Palerm-Viqueira, J. (2009): "Los comuneros regantes de la acequia real del río Júcar en Valencia, España". In T. Martínez-Saldaña, J. Palerm-Viqueira, M. Castro, \& L. Pereira (Eds.), Regadíos ancestrales en lberoamérica. Técnicas y organización social del pequeño riego. México: Mundi Prensa.

Puentes Poyatos, R., Velasco Gámez, M. D. M. \& Vilar Hernández, J. (2009): El buen gobierno corporativo en las sociedades cooperativas". REVESCO, 98 Segundo Cuatrimestre, pp 118-140. Retrieved from www.ucm.es/info/ revesco

Ruf, T. (2000): "Prefacio". In Antología sobre pequeño riego vol. II Organizaciones autogestivas, pp v-xii. México: Colegio de Postgraduados/ Plaza y Valdés.

RuF, T. (2011): "Dossier Le champ des commons en question: perspectives croisées. Le façonnage des institutions d'irrigation au XXe siècle, selon les principes d'Elinor Ostrom, est-il encore pertinent en 2010 ?", Natures Sciences Sociétés, 19, pp 395-404. 
RuIz, C. (2006): "Disfunciones en el gobierno de las sociedades cooperativas agrarias: el caso de las almazaras cooperativas". GEZKI, 2, pp 73-103. SAHLINS, M. (1977) [1974]: Economía de la edad de piedra. Madrid: Akal editor.

SIRRY, I. (1902): Irrigation in the Valley of the river Po Northern Italy. Being An Account Of A Mission Undertaken In The Summer Of 1899 For The Egyptian Government. Cairo: National Printing Department. Retrieved from https:// archive.org/details/cu31924022884427

SMITH, R. B. (1852): Italian Irrigation. Report on the agricultural canals of Piedmont and Lombardy, Vol 1 Historical and Descriptive. London/Edimburg: W. H. Allen and Co/W. Blacwood and Sons. Retrieved from https:// archive.org/details/italianirrigatiOOsmitgoog

$\mathrm{S}_{\text {MITH, }}$ R. B. (1855): Italian Irrigation. Report on the agricultural canals of Piedmont and Lombardy, Vol 2 Practical and Legislative (Second Edition.). Edinburg and London: W. Blackwood and Sons. Retrieved from https:// archive.org/details/italianirrigatiOOunkngoog

SOENS, T. (2011): "Floods and money: funding drainage and food control in coastal Flanders from the thirteenth to the sixteenth centuries". Continuity and Change, 26 (3), pp 333 - 365. doi:10.1017/S0268416011000221

SoEns, T. (2013): "Flood Security in the Medieval and Early Modern North Sea Area: A Question of Entitlement?". Environment and History, 19, pp 209-232. doi: 10.3197/096734013X13642082568651

SPEAR, R. (2004): "El gobierno democrático en las organizaciones cooperativas, CIRIEC-España". Revista de Economía Pública, Social y Cooperativa, 48, pp 11-30.

TeBRAKE, W. H. (2002): "Taming the waterwolf. Hydraulic engineering and water management in the netherlands during the middle ages". Tecnology and Culture, 43, pp 475-499.

TraWICK, P. (2001): "The Moral Economy of Water: Equity and Antiquity in the Andean Commons". American Anthropologist, 103 (2), pp 361-379.

TURNER II, B. L. (1981): "Agricultura y desarrollo del estado en las tierras bajas mayas". Estudios de Cultura Maya, XIII, pp 285-305. Retrieved from http:// www.revistas.unam.mx/index.php/ecm/article/download/35902/32648

VALE, J. (1905): "Irrigation of the Chen-tu Plain and Beyond". Journal of the China Branch of the Royal Asiatic Society, XXXVI, pp 36-50. Retrieved from e-asia.voregon.edu/zeasia/echina_v.htm

VIQueira, C. \& Palerm, A. (1954): "Alcoholismo, Brujería y Homicidio en Dos Comunidades Rurales de México". América Indígena, xiv (1), pp 7-36. 
VRIES, J. DE (1973): "On the Modernity of the Dutch Republic". The Journal of Economic History, 33 (1), pp 191-202.

WADE, R. (1988): Village Republics: economic conditions for collective action in south India. Cambridge University Press.

WHITE, L. (1973) [1962]: "La revolución agrícola en la alta edad media". In L. White, Tecnología medieval y cambio social. Buenos Aires: Editorial Paidós.

WiLmotт, W. E. (1989): "Dujiangyan: Irrigation and Society in Sichuan, China". The Australian Journal of Chinese Affairs, 22, pp 143-153.

Wittfogel, K. (1957): Oriental despotism. A comparative study of total power. New Haven and London: Yale University Press. Retrieved from http:// ia600702.us.archive.org/2/items/KarlAugustWittfogel-OrientalDespotism/ KarlAugustWittfogel-OrientalDespotism.pdf

Wittfogel, K. (1970): Agriculture: A Key to the Understanding of Chinese Society, Past and Present, The thirty.first George Ernest Morrison lecture in ethnology. Australian National University Press.

Wolf, E. (1957): "Closed corporate peasant communities in Mesoamerica and Central Java". Southwestern Journal of Anthropology, 13 (1), pp 1-18. Wolf, E. (1971) [1966]: Los Campesinos. Barcelona: Editorial Labor.

YongtanG, J. (1988): "Main experiences on design and management of the Dujiangyan irrigation system". Irrigation and Drainage Systems, 2, pp 173-184. 